\title{
Kernel Regression Residual Decomposition Method to Detect Rolling Element Bearing Faults
}

\author{
Xiaoqian Wang, ${ }^{1}$ Dali Sheng, ${ }^{2}$ Jinlian Deng $\mathbb{D},{ }^{3}$ Wei Zhang, ${ }^{3}$ Jie Cai, ${ }^{3}$ \\ Weisheng Zhao, ${ }^{3}$ and Jiawei Xiang $\mathbb{D}^{1}$ \\ ${ }^{1}$ College of Mechanical and Electrical Engineering, Wenzhou University, Wenzhou 325035, China \\ ${ }^{2}$ Department of Police Command and Tactics, Zhejiang Police College, Hangzhou 310053, China \\ ${ }^{3}$ Department of Mechanical Engineering, Zhejiang Institute of Mechanical \& Electrical Engineering, Hangzhou 310053, China
}

Correspondence should be addressed to Jinlian Deng; dengjinlian@zime.edu.cn and Jiawei Xiang; wxw8627@163.com

Received 29 January 2021; Revised 14 March 2021; Accepted 17 April 2021; Published 28 April 2021

Academic Editor: Zhifeng Dai

Copyright (c) 2021 Xiaoqian Wang et al. This is an open access article distributed under the Creative Commons Attribution License, which permits unrestricted use, distribution, and reproduction in any medium, provided the original work is properly cited.

\begin{abstract}
The raw vibration signal carries a great deal of information representing the mechanical equipment's health conditions. However, in the working condition, the vibration response signals of faulty components are often characterized by the presence of different kinds of impulses, and the corresponding fault features are always immersed in heavy noises. Therefore, signal denoising is one of the most important tasks in the fault detection of mechanical components. As a time-frequency signal processing technique without the support of the strictly mathematical theory, empirical mode decomposition (EMD) has been widely applied to detect faults in mechanical systems. Kernel regression (KR) is a well-known nonparametric mathematical tool to construct a prediction model with good performance. Inspired by the basic idea of EMD, a new kernel regression residual decomposition (KRRD) method is proposed. Nonparametric Nadaraya-Watson KR and a standard deviation (SD) criterion are employed to generate a deep cascading framework including a series of high-frequency terms denoted by residual signals and a final low-frequency term represented by kernel regression signal. The soft thresholding technique is then applied to each residual signal to suppress noises. To illustrate the feasibility and the performance of the KRRD method, a numerical simulation and the faulty rolling element bearings of well-known open access data as well as the experimental investigations of the machinery simulator are performed. The fault detection results show that the proposed method enables the recognition of faults in mechanical systems. It is expected that the KRRD method might have a similar application prospect of EMD.
\end{abstract}

\section{Introduction}

The device of rotating machinery is entirely dependent on the health condition of the rolling element bearings, which accounts for almost $40-50 \%$ of these equipment failures $[1,2]$. Such failures could be disastrous or could lead to the shutdown of the entire production lines, potentially causing huge losses [3]. Therefore, fault detection of bearing has been a great challenge in recent decades [4]. In order to obtain the fault feature, the vibration signal is one of the most important sources of information for monitoring conditions of rolling element bearing. However, the vibration signals are often contaminated by various noises, and sometimes, it is difficult to obtain high-fidelity signals. Therefore, signal denoising is one of the significant tasks to detect faults in rolling element bearings [5].

In the past few decades, a lot of novelty approaches have been proposed [6-8]. Time-frequency (TF) method techniques have been developed to allow access to the timefrequency energy behavior of nonstationary signals. TF representations can give insight into the complex structure of signals consisting of several components [9]. It maps a 1D signal to a $2 \mathrm{D}$ function of time and frequency, so a TF representation can be obtained to characterize the signal in the time and frequency domains simultaneously [10]. Many signal time-frequency methods were applied to machine 
diagnostics in the last few decades. The wavelet decomposition method has been most commonly used [11-14], and an arbitrary signal can be decomposed into a set of wavelet coefficients. At each scale, the soft thresholding technique is performed to suppress noises, and finally, the wavelet reconstructed technique is employed to obtain the purified signals. Lei et al. [15] presented a method based on wavelet packets and Hilbert-Huang transform to improve productivity and part quality in the machining process. A remarkable advantage of the wavelet transform method is that the wavelet transform is sensitive to defects for a longer duration reflected by nonstationary signals [6]. However, wavelet transform suffers from disadvantages, one limitation worth noting that the usage of wavelet transforms to suppress noise will often lead to oscillation effects when dealing with the low signal-to-noise ratio (SNR) scenarios [16]. And another challenge is to select the appropriate wavelets [17-19].

Empirical mode decomposition (EMD) is one of the most powerful time-frequency analysis techniques proposed by Huang [18], which has been widely applied to detect faults in rotating machinery $[19,20]$. EMD is a self-adaptive signal processing method that could be used in nonlinear and nonstationary process; it decomposes the complicated signal into intrinsic mode functions (IMFs). Frequency components contained in each IMF not only relate to the sampling frequency but also change with the signal itself [21]. Therefore, using EMD combine with the Hilbert spectrum analysis to reveal the faulty frequency information hidden in vibration signals, the result shows good computational efficiency and resolution in nonlinear and nonstationary process. Recently, ensemble empirical mode decomposition (EEMD) is developed by $\mathrm{Wu}$ and Huang [22]. EEMD is a noise-assisted data analysis method, and by adding finite white noise to the investigated signal, the EEMD method can eliminate the mode mixing problem in all cases automatically [23]. Lei et al.[24, 25] used EEMD to enhance the efficiency of the feature extraction of faulty signals as well as decrease the mode mixing phenomenon. In working conditions, the key issues are how the EMD worked with the support of the strictly mathematical theory and how to properly select the added noise amplitude $[26,27]$.

Kernel regression (KR) $[28,29]$ is a nonparametric technique to construct a prediction model based on Nadaraya-Watson kernel estimator $[30,31]$. In recent years, $\mathrm{Wu}$ et al.[32,33] presented two hybrid approaches using the KR technique and other methods to predict faults in car assembly line, e.g., the fuzzy wavelet kernel support vector classifier machine and modified genetic algorithm, and the triangular fuzzy Gaussian wavelet kernel support vector classifier machine and genetic algorithm. Baraldi et al. [34] presented a modification of the traditional autoassociative kernel regression (AAKR) method which enhances the signal reconstruction robustness so as to monitor the abnormal conditions of industrial components. In [35], kernel regression method is used in image denoising, and results illustrate good performance in color estimates. Compared to the EMD method without the support of the strictly mathematical theory, $\mathrm{KR}$ is a nonparametric mathematical tool based on statistical mathematical theory. However, the above methods are only the usage of the prediction characteristics of $\mathrm{KR}$. Therefore, it might be interesting to generate a methodology like EMD to decompose vibration signals, i.e., a new kernel regression residual decomposition (KRRD) method. Besides, the standard deviation (SD) criterion [18] can be applied to automatically determine the decomposition level of the KRRD algorithm. The KRRD algorithm is also reversible using kernel regression residual reconstitution (KRRR) algorithm.

According to the multiscale signal decomposition principle, the well-known soft threshold technique proposed by Donoho $[12,36]$ can be applied to denoise signals at each scale.

The rest of the paper is organized as follows. Brief introductions of the basic idea of the KRRD algorithm, SD criterion, soft threshold technique, and KRRR algorithm are given in Section 2. In Section 3, a numerical simulation and an open-access faulty bearing data are carried out to validate the effectiveness of the present method, and the experimental investigations for three cases are further given in Section 4. Conclusions and further work are drawn in Section 5 .

\section{Kernel Regression Residual Decomposition Methodology}

Regression model-based methods will be applied to decompose signals, according to the basic idea of EMD. In the present, $\mathrm{KR}$ is employed as a bridge to form an explicit KRRD method. Figure 1 shows the three steps of the proposed KRRD. Firstly, the residual signals of a raw signal are obtained using KR repeatedly, which can be called KRRD. Secondly, the soft thresholding technique and SD criterion are applied to obtain noise-suppressed residual signals. KRRR is then used to reconstruct the purified signal with high SNR. Finally, to obtain demodulation frequencies, the traditional Hilbert envelope spectrum analysis is applied in the paper and the fault types will be given.

2.1. KRRD and KRRR. The procedure of KRRD is simply listed as follows. The kernel regression method has been most commonly used; a signal $f(t)$ can be represented by the summation of $n$ residual signals as

$$
f(t)=\sum_{i=1}^{n} r_{i}(t)=f \sum, 1(t)
$$

where $r_{i}(t)(i=1,2, \ldots, n)$ is a residual signal characterizing the signal information at scale $i$ and $f \sum, 1(t)$ is the summation of all residual signals at scale $[1, n]$, i.e., the original/ raw signal $f(t)$. In order to decompose the signal $f(t)$ into a number of residual signals, a deep framework can be rewritten by

$$
f \sum, 1(t)=f \sum, 2(t)+r_{1}(t)=s_{1},
$$

where $f \sum, 2(t)$ denote the summation of all residual signals at scale $[2, n]$. In this step, estimating $f \sum, 2(t)$ by given 


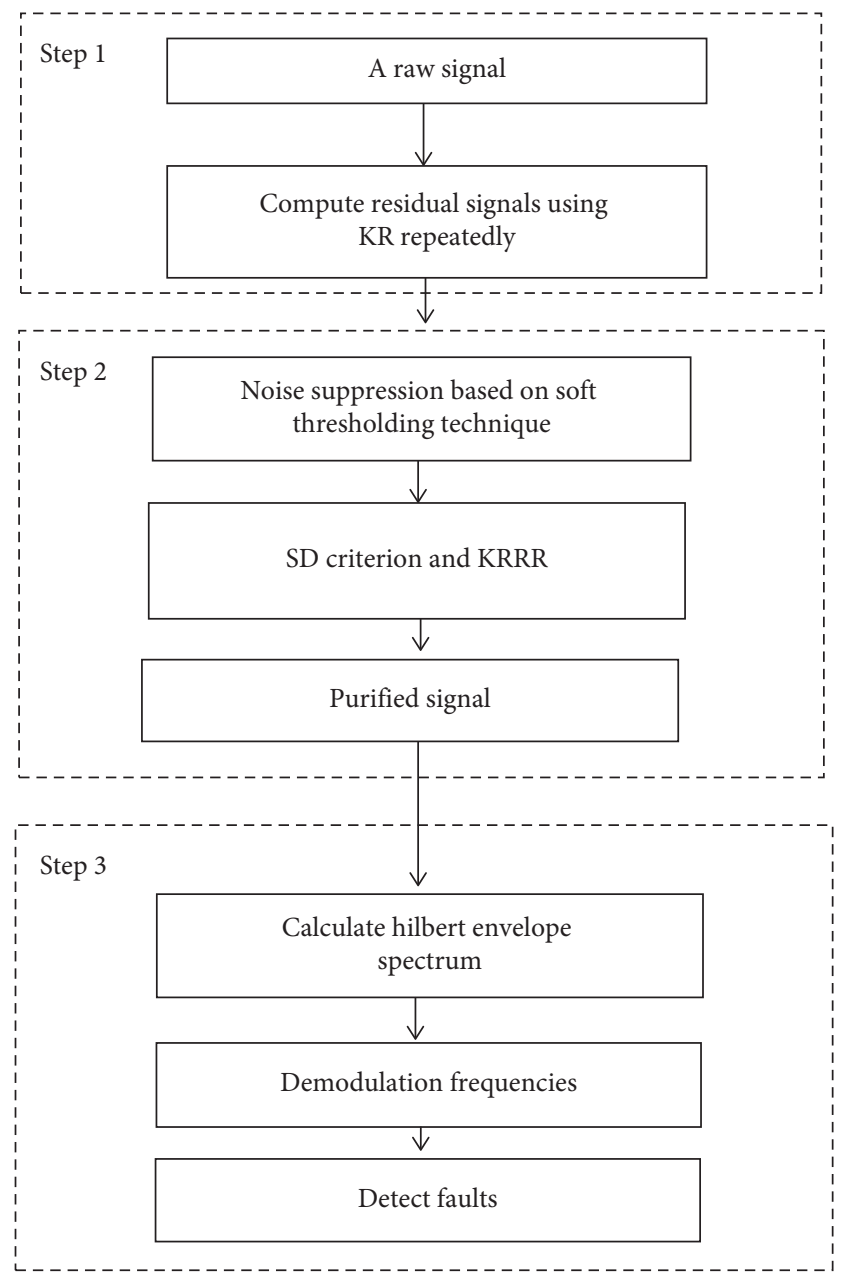

FIgURE 1: Flowchart of the proposed KRRD method.

$f \sum, 1(t)$ is the key to solve the problem. In the present, a KR strategy is used in conditional expectation. Therefore, the estimation of $f \sum, 2(t)$ can be represented by $f \sum, 1(t)$ as

$$
f \sum, 2(t)=K_{1}\left(f \sum, 1(t)-f \sum, 1\left(t_{i}\right)\right)=s_{2},
$$

where the 2nd smooth term $s_{2}=K_{1}\left(f \sum, 1(t)-f \sum, 1\left(t_{i}\right)\right)$ can be estimated by nonparametric Nadaraya-Watson KR $[29,30]$ and $K$ is a density function. In the present, a radial basis function (RBF) kernel [37] $K_{1}$ is employed and $f \sum, 2(t)$ can be obtained by

$$
f \sum, 2(t)=K_{1}\left(f \sum, 1(t)-f \sum, 1\left(t_{i}\right)\right)=e^{-\frac{1}{2 \lambda_{1}^{2}}\left(f \sum, 1(t)-f \sum, 1\left(t_{i}\right)\right)^{2}},
$$

where $\lambda_{1}$ is the bandwidth of the $f(t)$ and $f \sum, 1(t)$ is the Gaussian kernel function center. It is worth pointing out that the RBF has the characteristic of unique best approximation to the unknown time series [37]. Since the residual signal at scale $n$ can be obtained by equation (4), a deep cascading framework can be expressed as follows:

$$
r_{j}(t)=f \sum, j(t)-f \sum, j+1(t)=s_{j}-s_{j+1},
$$

in which the $(j+1)$ th smooth term $\mathrm{s}_{j+1}=K_{j}\left(f \sum, j+1(t)-\right.$ $\left.f \sum, j+1\left(t_{i}\right)\right)$ can also be estimated by nonparametric
Nadaraya-Watson KR. The last residual signal can be expressed as follows:

$$
r_{n}(t)=s_{n} .
$$

Finally, the signal decomposed realization of KRRD via kernel regression at each scale is completed, and the KRRD is simply the summation of all residual signals $r_{1}(t), r_{2}(t), \ldots, r_{n}(t)$ which is shown in Figure 2. Besides, the residual signals at the lower scales contain great characteristics of the signal, while the signal contained coarser signal characteristics. 


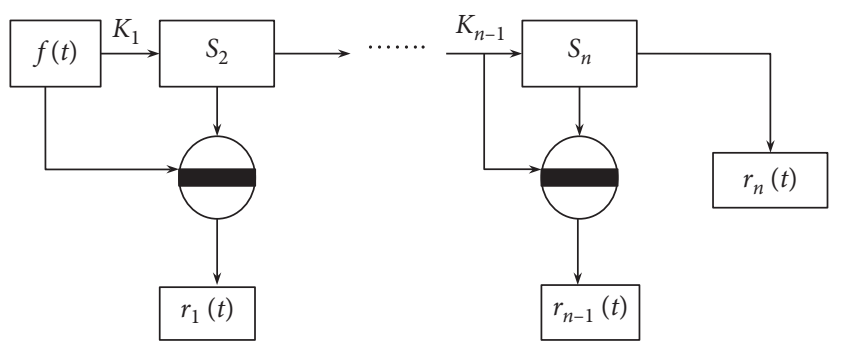

Figure 2: The framework of KRRD.

2.2. Noise Suppression. In this paper, the work is to illustrate the feasibility of utilizing the KRRD for processing fault signals. However, at each scale, there is some low but not useful information. The soft thresholding technique [12, 36] is the commonly used tool to obtain greater content at each scale.

Since residual signals at different scales are obtained. Considering some unusual data in the signal, we use the median absolute deviation function to estimate the noise threshold $\theta_{j}$ at scale $j$, using adaptive noise estimate. The threshold strategy can be expressed as follows:

$$
\theta_{j}=\frac{\operatorname{MAD}\left(r_{j}\right)}{0.6745}
$$

where MAD is the median absolute deviation and 0.4675 is the normal inverse cumulative distribution function of $3 / 4$ [38]. Based on soft thresholding, $C_{j}(t)$ can be obtained by

$$
C_{j}(t)= \begin{cases}0, & \text { if }\left|r_{j}(t)\right|<\theta_{j} \\ \left|r_{j}(t)\right|-\theta_{j}, & \text { otherwise }\end{cases}
$$

The sifting usually has to be implemented more times, but to get the most useful information and improve the computational efficiency of this method. In practice, we used the standard deviation (SD) criterion [39] based on the EMD method to determine whether or not $C_{j}(t)$ well satisfies the IMF properties; the SD can be expressed as follows:

$$
S D(k)=\sum_{t=0}^{T}\left[\frac{\left|C_{j-1}(t)-C_{j}(t)\right|^{2}}{C_{j-1}^{2}(t)}\right],
$$

where $T$ is the length of data. This procedure should be repeatedly used for $n$ times until the last residue $C_{\mathrm{n}}(t)$ satisfies the formula. In KRRD, the process of calculation is usually set to 0.2 , and if SD is less than the threshold, the process stops and the decomposition procedure is finished.

Since the circulated shift is invertible, the reverse shift is simply the summation of all residual signals, which can be represented by

$$
f(t)=R^{-1}\left(C_{1}(t), C_{2}(t), \ldots, C_{n}(t)\right)=\sum_{i=1}^{n} C_{i}(t) .
$$

The framework of KRRR is shown in Figure 3. Finally, the inverse KRRD called KRRR is used to reconstruct the new purified signal.

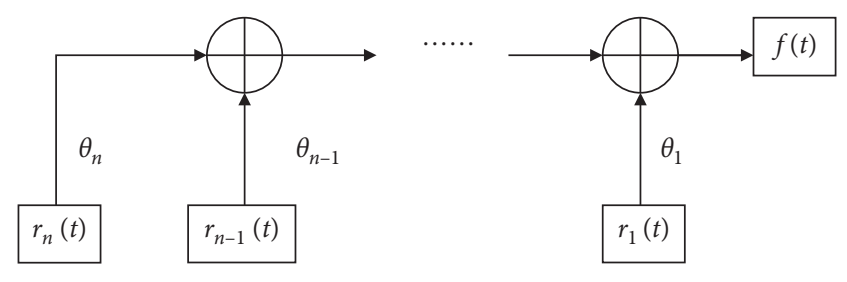

FiguRE 3: The framework of KRRR.

2.3. Fault Detection. The traditional Hilbert envelope spectrum analysis $[18,40]$ is then applied to determine demodulation frequencies.

Compared the theoretical feature frequencies of faults in bearings with demodulation frequencies, the type of bearing faults could finally be determined.

\section{Simulation Analysis}

In this section, to validate the effectiveness of the proposed method, a simulated signal which contains random noise is constructed [41, 42]. The model of the signal is expressed as follows:

$$
f(t)=x(t)+R(t)
$$

where $x(t)$ is the periodic impulse signal without noise, $R(t)$ is the random noise, and $f(t)$ is the simulation signal. Suppose the sampling frequency is $20 k$ and the sampling points are 8192 , the impulse signal and the noise-contaminated signal are shown in Figures 4(a) and 4(b), respectively. It is clear to see that the signal is immersed in heavy noises.

The decomposition signals using KRRD are shown in Figures 4(c)-4(e). As shown in Figures 4(c)-4(e), the KRRD method can effectively distinguish the narrow band impulses (see Figures 4(c)-4(e)) from the purified signal (see Figure $4(\mathrm{f}))$. It can also be observed that the noise process is well characterized at the low scales and the structural characteristics of the simulation signal are well characterized as the scale $j$ increases. It indicates that the proposed method can be regarded as a promising method to decompose signals. To further clarify the denoising process of the KRRD method, the faulty bearing data of Case Western Reserve University [43] are used in this paper. The proposed method is applied to detect the bearing with inner race fault, the sampling frequency $f_{s}$ is set to $25.6 \mathrm{kHz}$, the collected data length is 12288 points, the shaft rotating frequency is $f_{r}=29.5 \mathrm{~Hz}$, and bearing pass frequency of inner race (BPFI) is $159.93 \mathrm{~Hz}$. The bearing fault data are added with Gaussian noises for SNR $=4 \mathrm{~dB}$ to illustrate the effectiveness of this method and the results are shown in Figure 5.

From Figure 5(a), the raw signal $S_{1}$ is divided into four residual signals using kernel regression function. By comparing with Figure 5(b), it can be observed that in Figure 5(b), it is clear that each residual signal is decomposed using noise suppression.

The raw signal and the corresponding denoising signal are shown in Figure 6. And the frequency spectrum of raw signal and denoising signal can be seen in Figure 7. It is 


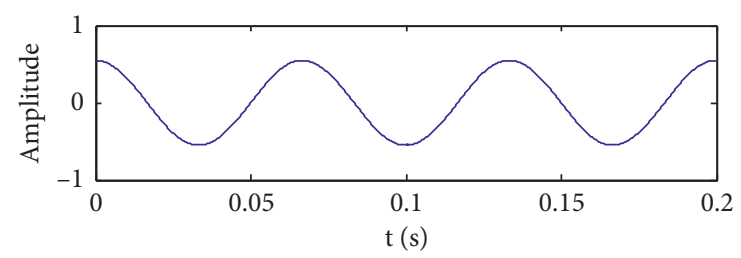

(a)

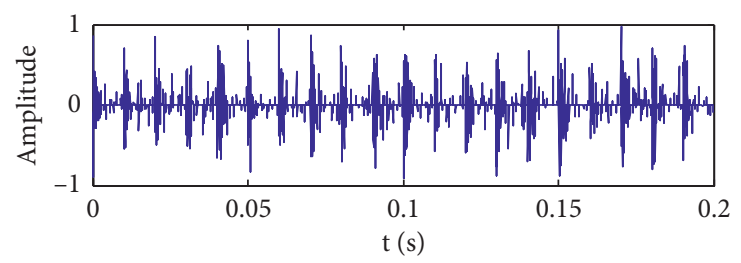

(c)

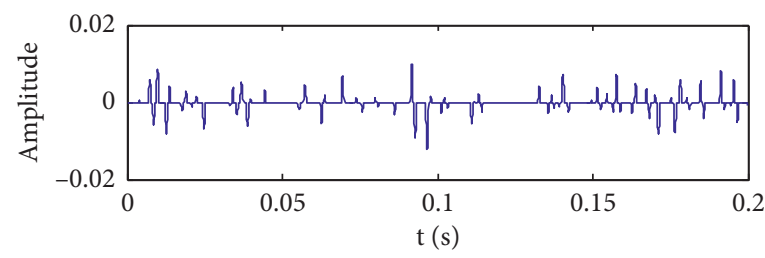

(e)

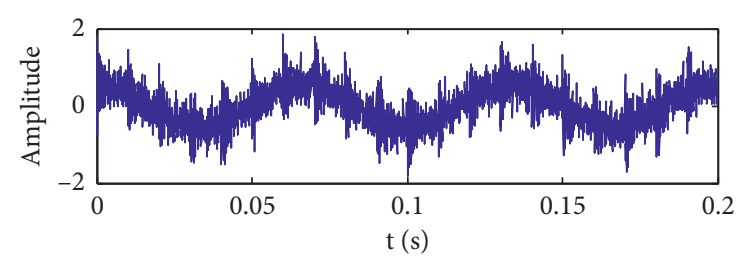

(b)

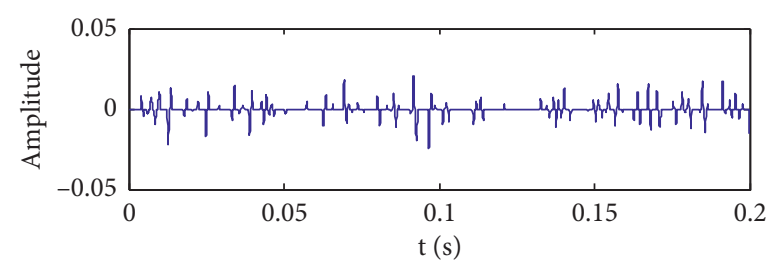

(d)

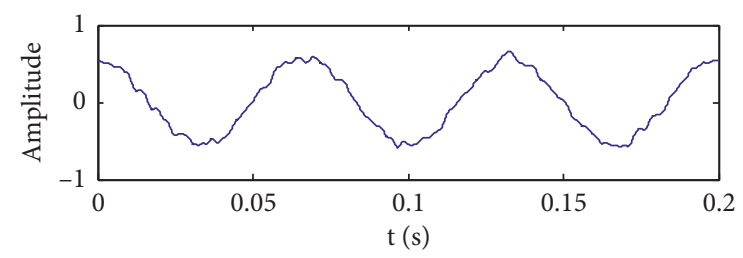

(f)

Figure 4: The simulation signal. (a) Test signal. (b) Noisy input signal. (c-e) Decomposition signals. (f) Purified signal.

worth noting that the fault frequency of $154.2 \mathrm{~Hz}$ is shown in Figure 7(b), which is more accurate than Figure 7(a) and the fault frequency of $154.2 \mathrm{~Hz}$ is matching with the theoretical calculation value of $159.93 \mathrm{~Hz}$. Based on the above investigation, we conclude that the present approach can be employed to determine the faults of bearings.

\section{Experimental Evaluation}

In this section, the proposed approach is evaluated by rolling element bearings with inner race, outer race, and compound fault $[12,44]$. Generally, the vibration response of a bearing with faults consists of a series of impulses, whereas the frequencies in these impulses are the bearing characteristic frequencies (BCFs). Figure 8 shows the defects at the outer race, inner race, and ball. It is noted that a deep groove bearing (ER-12K) is used in the experiment [45]. Three fault types in the present experimental investigations are bearing pass frequency of outer race (BPFO), bearing pass frequency of inner race (BPFI), and ball spin frequency (BSF) [2], which can be theoretically calculated by

$$
\begin{aligned}
\mathrm{BPFO} & =\frac{n f_{r}}{2}\left[1-\frac{d}{D} \cos \phi\right], \\
\mathrm{BPFI} & =\frac{n f_{r}}{2}\left[1+\frac{d}{D} \cos \phi\right], \\
\mathrm{BSF} & =\frac{D f_{r}}{2 d}\left[1-\left(\frac{d}{D} \cos \phi\right)\right],
\end{aligned}
$$

in which $f_{r}$ is the shaft speed $(\mathrm{Hz}), n$ is the number of rolling elements, $\phi$ is the angle of the load from the radial plane, $d$ is the ball diameter, and $D$ is the pitch diameter.

The fault simulator (MFS-MG, manufactured by Spectraquest Inc.) and the test system are shown in Figure 9. The test system includes speed indicator, manual speed governor, acceleration sensors, speed sensor, motor, spindle, bearings, etc. The bearing parameters are the number of rolling elements $n=8$, ball diameter $d=0.3125$ inches, pitch diameter $D=1.318$ inches, and the contact angle $\phi=0^{\circ}$. During the experiment, the data are acquired by an accelerometer mounted on the top of the bearing holder on the left side, and the sampling frequency $f_{s}$ is set to $25.6 \mathrm{kHz}$.

4.1. Outer Race Fault Detection. In this section, the proposed method is applied to detect the bearing with outer race fault. For the bearing with outer race fault, the collected data length is 12288 points and the shaft rotating frequency is $f_{r}=29.87 \mathrm{~Hz}$. According to equation (12), BPFO can be calculated as

$$
\mathrm{BPFO}=\frac{n f_{r}}{2}\left[1-\frac{d}{D} \cos \phi\right]=91.15 \mathrm{~Hz} .
$$

From Figure 10(a), it can be seen that, due to the defect present in the rolling bearing, the vibration signal presents the impacts feature, but there exist very serious noises. Applying the KRRD method to the raw signal, the denoised signal is shown in Figure 10(b). By comparing with Figure $10(\mathrm{a})$, the ambient noises are effectively suppressed. The fault characteristic frequency $(87.5 \mathrm{~Hz})$ is clearly revealed. The comparisons show that the above method 

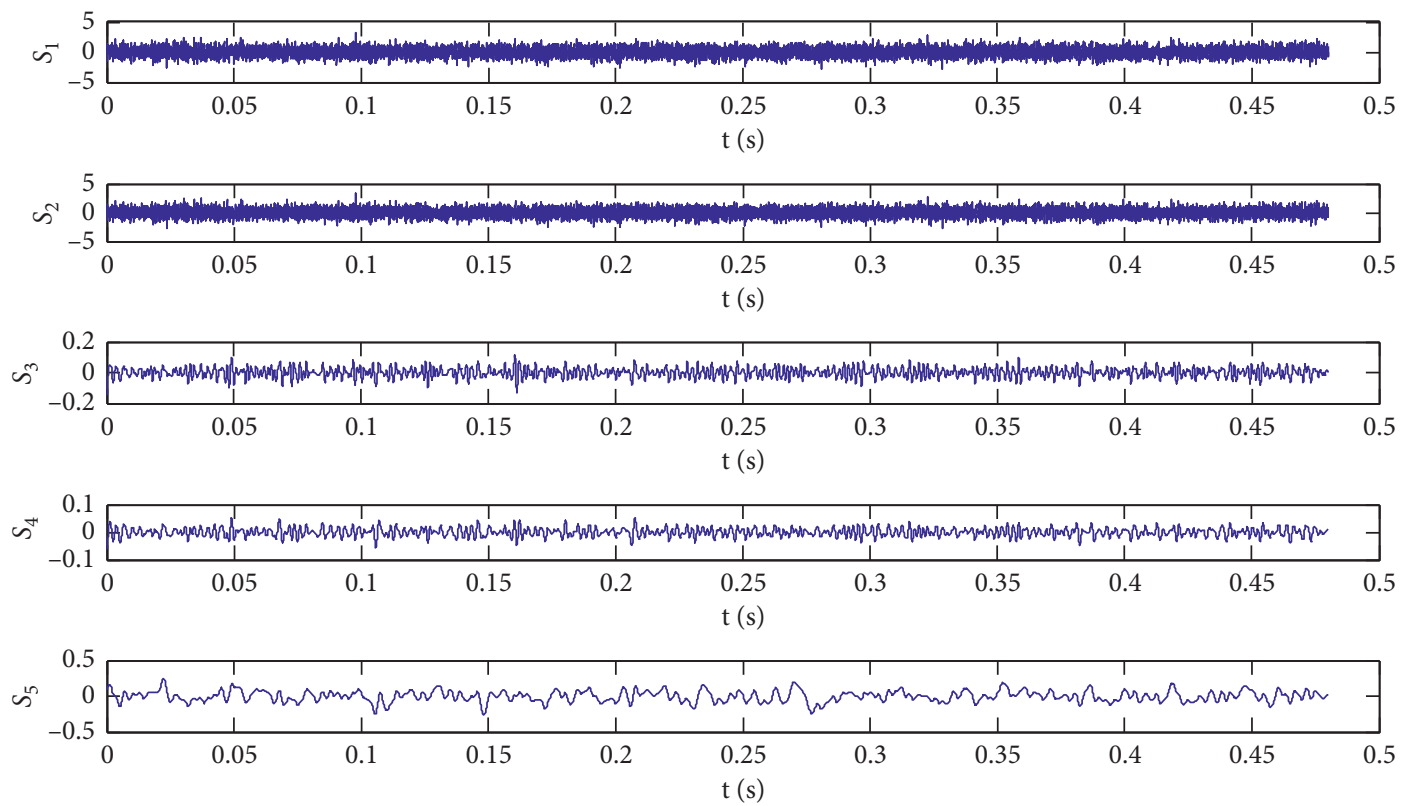

(a)
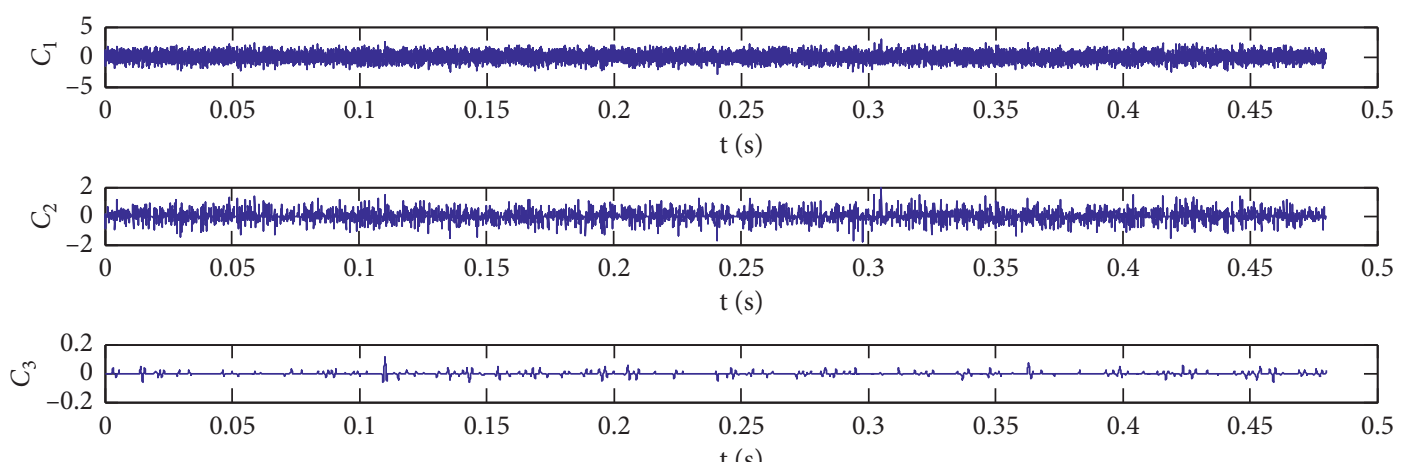

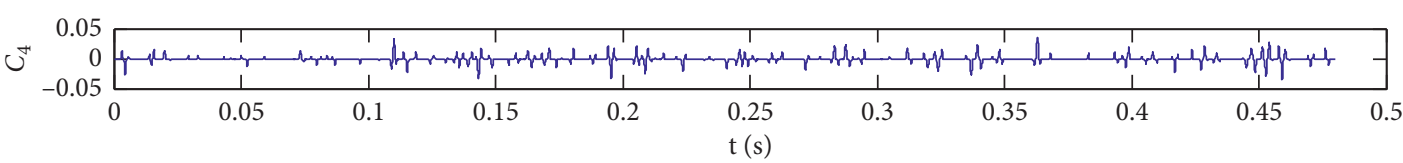

$\sim_{0}^{n} \underbrace{0.2}_{0.05}$

(b)

FIgURE 5: The KRRD method using Case Western Reserve University data. (a) Raw signal $S_{1}$ is decomposed into four residual signals $S_{2}$, $S_{3}$, $S_{4}$, and $S_{5}$. (b) Each residual signal is decomposed into $C_{1}, C_{2}, C_{3}, C_{4}$, and $C_{5}$ using noise suppression.

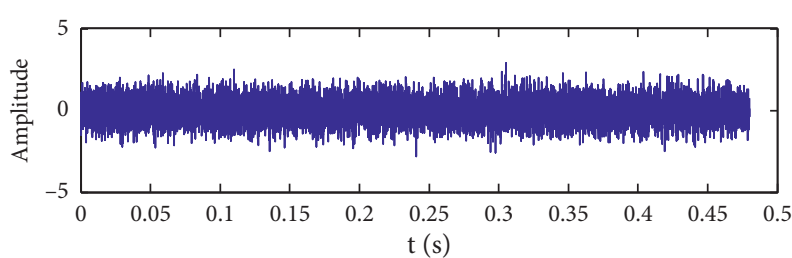

(a)

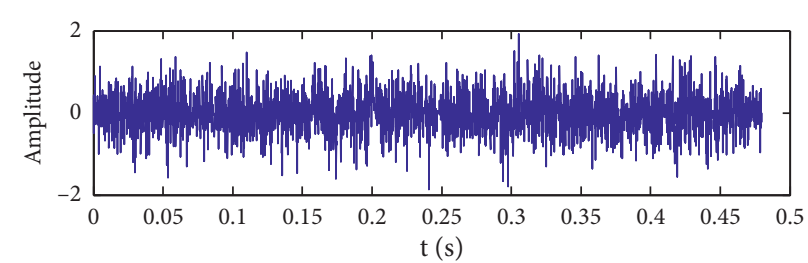

(b)

FIgURE 6: The KRRD method using Case Western Reserve University data. (a) Raw signal. (b) Denoising signal. 


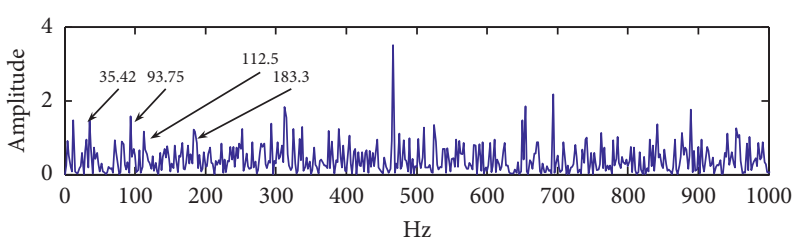

(a)

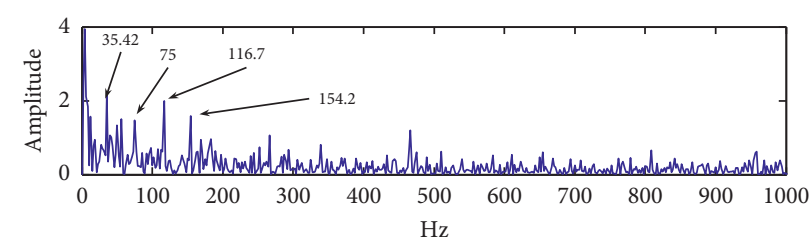

(b)

Figure 7: The frequency spectrum of Case Western Reserve University data. (a) The frequency spectrum of the raw signal. (b) The frequency spectrum of the denoising signal.

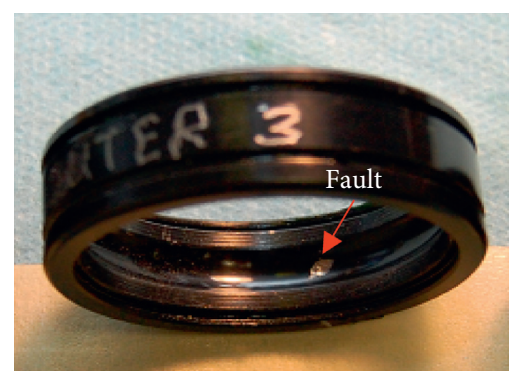

(a)

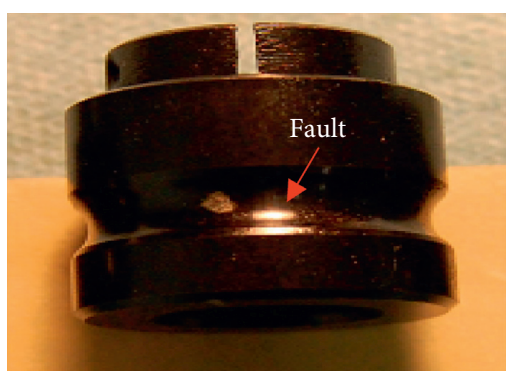

(b)

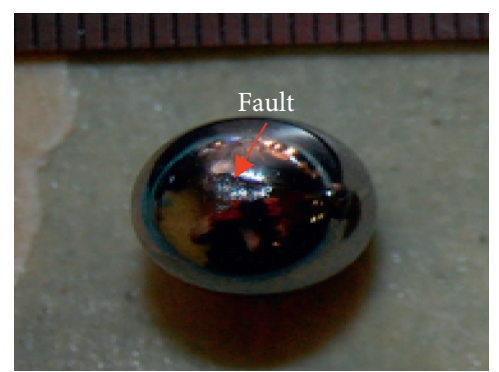

(c)

Figure 8: The faults in the bearing. (a) The fault in the outer race of the bearing. (b) The fault in the inner race of the bearing. (c) The fault in the ball of the bearing.

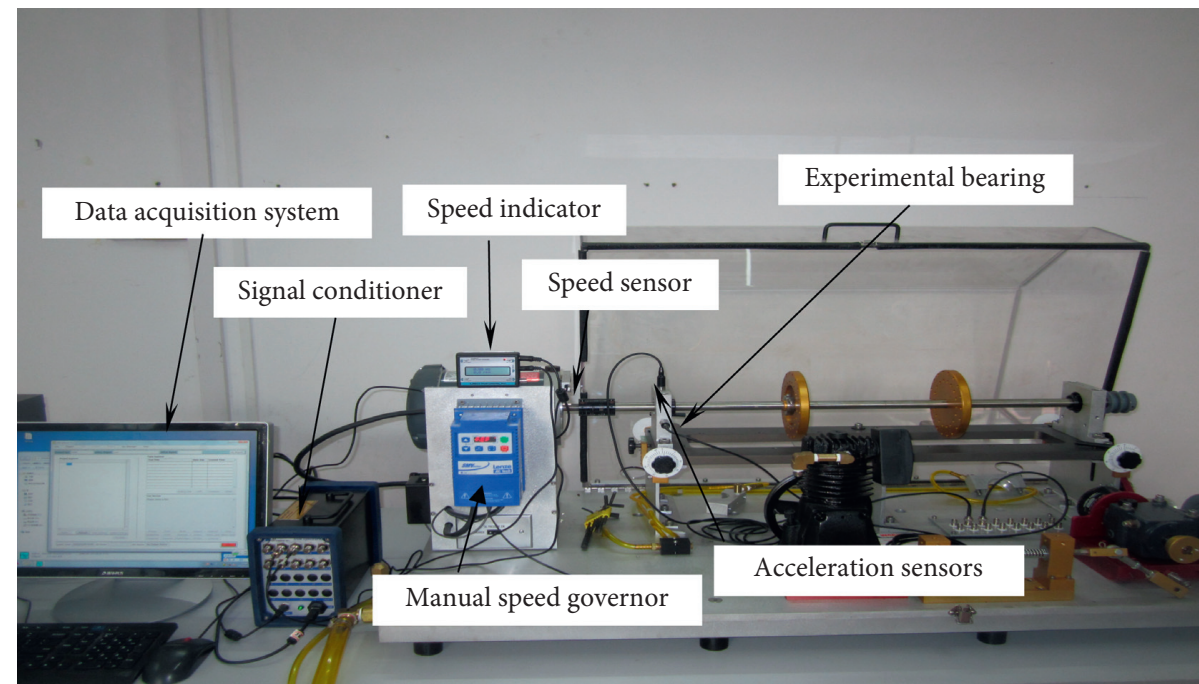

FIGURE 9: The machinery fault simulator.

proposed in this paper is more effective. Therefore, the bearing with outer race fault will be clearly detected.

4.2. Inner Race Fault Detection. For the bearing with inner race fault, the total collected data are 12288 points; the shaft rotating frequency is $f_{r}=29.87 \mathrm{~Hz}$. The ball pass frequency of the outer race $(\mathrm{BPFI})$ is given by

$$
\mathrm{BPFI}=\frac{n f_{r}}{2}\left[1+\frac{d}{D} \cos \phi\right]=147.8 \mathrm{~Hz} .
$$

In this section, the proposed method is applied to detect the bearing with inner race fault. By comparing Figures 11(a) and 11(b), we can see that the impact characteristic is not clearly shown in the former graphic but clearly shown in the latter graphic. And the fault frequency of the inner race in 

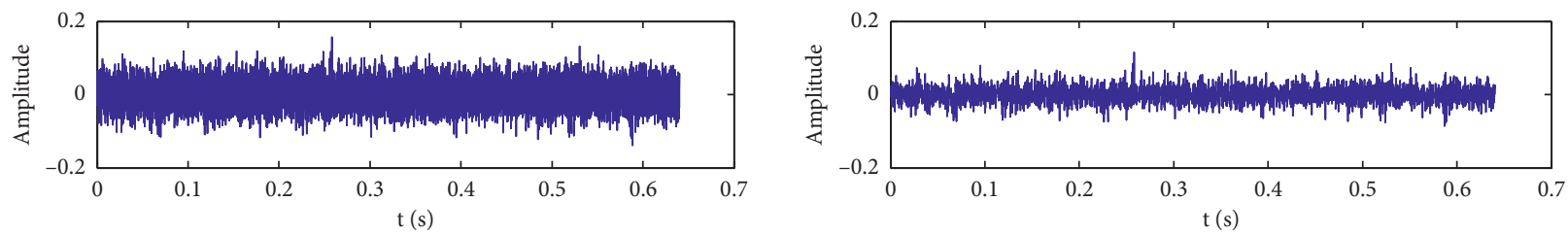

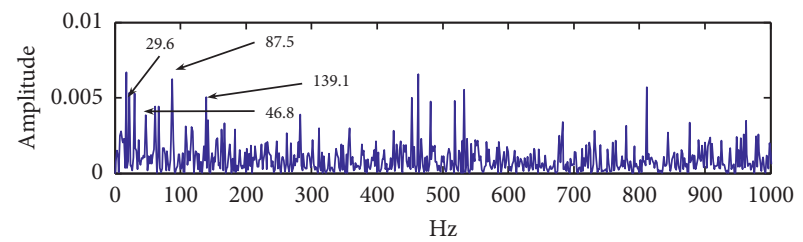

(a)

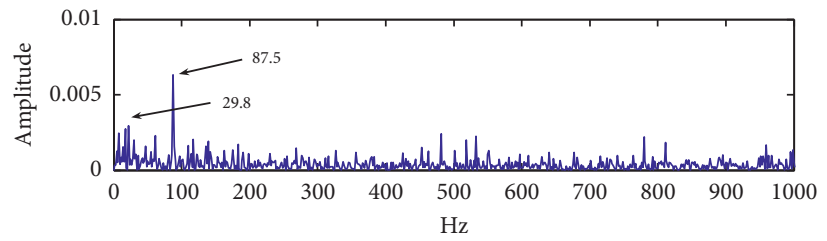

(b)

FIgURE 10: The outer race experiment signal. (a) The raw signal and the frequency spectrum of the raw signal. (b) The denoised signal and the frequency spectrum of the denoised signal.
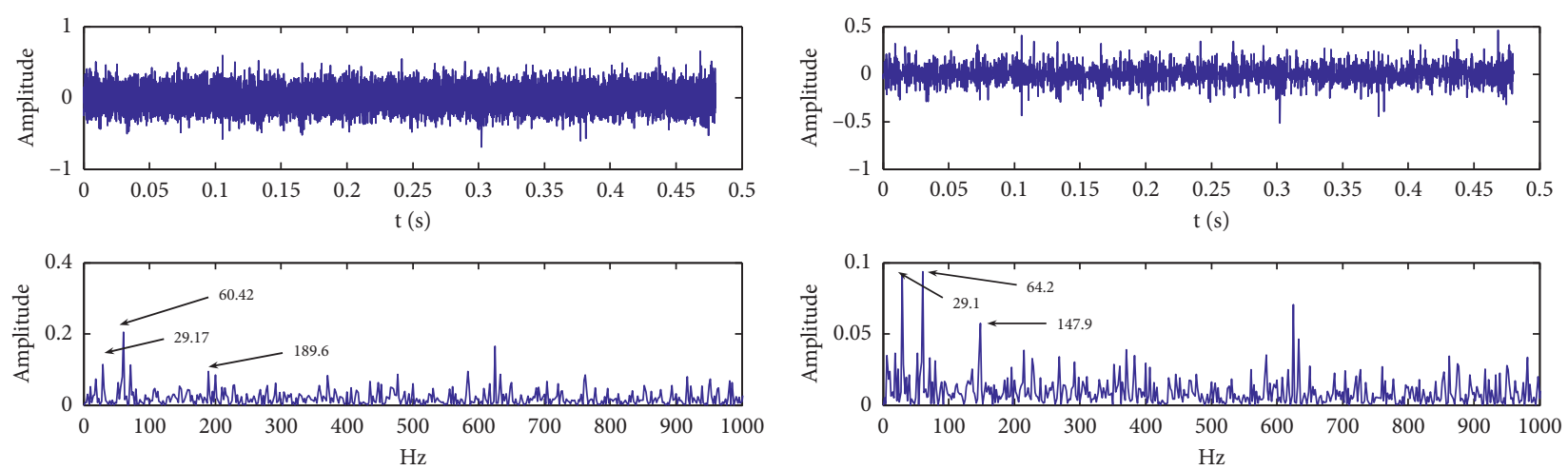

(a)

(b)

Figure 11: The inner race experiment signal. (a) The raw signal and the frequency spectrum of the raw signal. (b) The denoised signal and the frequency spectrum of the denoised signal.

Figure 11(a) is not clearly identified, but in Figure 11(b), the shaft rotating frequency is $29.87 \mathrm{~Hz}$, second harmonic frequency is $59.26 \mathrm{~Hz}$, and its feature frequency of $147.9 \mathrm{~Hz}$ is matching with the theoretical calculation value of $147.8 \mathrm{~Hz}$, and we conclude that there is a fault in inner race of the bearing.

4.3. Compound Fault Detection. For the bearing with compound fault (the combination of BPFO and BPFI), the total collected data are 12288 points, the shaft rotating frequency is $f_{r}=39.82 \mathrm{~Hz}$, and the corresponding BPFO is given by

$$
\mathrm{BPFO}=\frac{n f_{r}}{2}\left[1-\frac{d}{D} \cos \phi\right]=121.5 \mathrm{~Hz} .
$$
by

The bearing pass frequency of inner race (BPFI) is given

$$
B P F I=\frac{n f_{r}}{2}\left[1+\frac{d}{D} \cos \phi\right]=197.1 \mathrm{~Hz} .
$$

The ball spin frequency (BSF) is given by

$$
\mathrm{BSF}=\frac{D f_{r}}{2 d}\left[1-\left(\frac{d}{D} \cos \phi\right)^{2}\right]=79.3 \mathrm{~Hz}
$$

In this section, the proposed method is applied to detect the bearing with compound fault. The original signal is illustrated in Figure 12(a) and the denoised signal using the KRRD method is shown in Figure 12(b). In Figure 12(a), only the input shafting frequency of $40 \mathrm{~Hz}$ is shown, while the other 

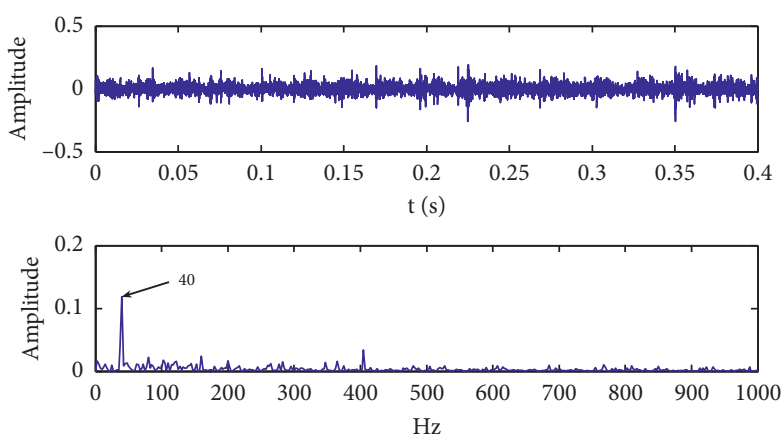

(a)
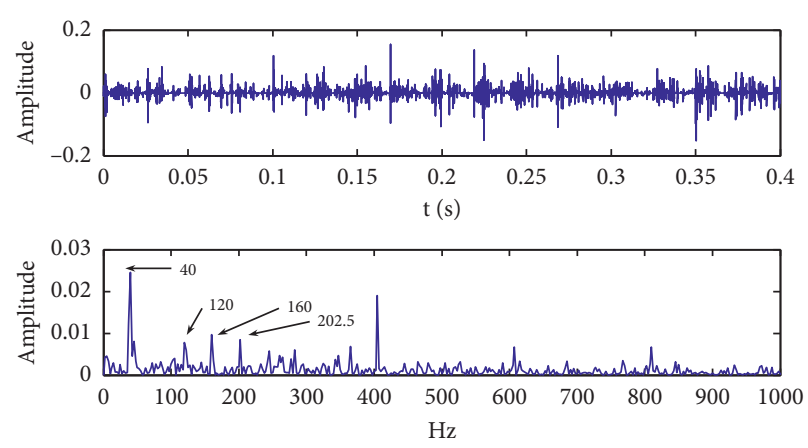

(b)

Figure 12: The compound fault experiment signal. (a) The raw signal and the frequency spectrum of the raw signal. (b) The denoised signal and the frequency spectrum of the denoised signal.

harmonics are submerged by other unknown frequency components; it is not clear what kind of fault is. From Figure 12(b), the input shafting rotating frequency $(40 \mathrm{~Hz})$ and its inner race fault $(202.5 \mathrm{~Hz})$, outer race fault $(120 \mathrm{~Hz})$, and the second harmonic frequency of ball fault $(79.25 \mathrm{~Hz})$ are matching with the theoretical calculation values. The results demonstrate that the proposed approach can effectively extract the fault features of defective bearings.

\section{Conclusion}

In this paper, aiming at the shortcoming of the conventional denoising method for bearing fault signal under variation conditions, a new KRRD method is proposed. Like the EMD method, the KRRD method is used to decompose a signal into a number of residual signals at different scales. It allows the noise suppression method to get rid of the noise information while preserving the important signal characteristics. The method is verified by the feature extraction of the faulty bearing of the outer race, inner race, and compound fault. From the detection results of the numerical simulation and experiment investigations from both open access data and mechanical fault simulator, it can be observed that the present method is suitable to detect faults in the mechanical systems. Because KR is a nonparametric mathematical tool with the support of the strictly mathematical theory, KRRD might be superior to EMD. The decomposition procedures are similar to EMD, and the application area can be enlarged if more research works are further performed. Moreover, it is expected that the procedures of the present method are surely suitable to other regression models.

\section{Data Availability}

The data used to support the findings of this study are available on the Case Western Reserve University Bearing Data Center website (http://csegroups.case.edu/bearingdatacenter/home).

\section{Conflicts of Interest}

The authors declare that they have no conflicts of interest.

\section{Acknowledgments}

The authors acknowledge the support received from the Zhejiang Provincial Key Research and Development Project (grant no. 2020C0309) and Zhejiang Provincial Science Foundation (grant no. GF19F020010).

\section{References}

[1] S. Nandi, H. A. Toliyat, and X. Li, "Condition monitoring and fault diagnosis of electrical motors-a review," IEEE Transactions on Energy Conversion, vol. 20, no. 4, pp. 719-729, 2005.

[2] R. B. Randall and J. Antoni, "Rolling element bearing diagnostics-A tutorial," Mechanical Systems and Signal Processing, vol. 25, no. 2, pp. 485-520, 2011.

[3] S. Wang, J. Xiang, H. Tang, X. Liu, and Y. Zhong, "Minimum entropy deconvolution based on simulation-determined band pass filter to detect faults in axial piston pump bearings," ISA Transactions, vol. 88, pp. 186-198, 2019.

[4] A. Kumar, C. P. Gandhi, Y. Zhou, R. Kumar, and J. Xiang, "Latest developments in gear defect diagnosis and prognosis: a review," Measurement, vol. 158, Article ID 107735, 2020.

[5] S. Wang, J. Xiang, Y. Zhong, and H. Tang, "A data indicatorbased deep belief networks to detect multiple faults in axial piston pumps," Mechanical Systems and Signal Processing, vol. 112, pp. 154-170, 2018.

[6] A. Kumar, Y. Zhou, and J. Xiang, "Optimization of VMD using kernel-based mutual information for the extraction of weak features to detect bearing defects," Measurement, vol. 168, Article ID 108402, 2021.

[7] J. Urbanek, T. Barszcz, M. Strączkiewicz, and A. Jablonski, "Normalization of vibration signals generated under highly varying speed and load with application to signal separation," Mechanical Systems and Signal Processing, vol. 82, pp. 13-31, 2017.

[8] Y. Gao, X. Liu, and J. Xiang, "FEM simulation-based generative adversarial networks to detect bearing faults," IEEE Transactions on Industrial Informatics, vol. 16, no. 7, pp. 4961-4971, 2020.

[9] L. Cohen, "Time-frequency distributions-a review," Proceedings of the IEEE, vol. 77, no. 7, pp. 941-981, 1989.

[10] Y. Yang, Z. Peng, X. Dong, W. Zhang, and G. Meng, “Application of parameterized time-frequency analysis on multicomponent frequency modulated signals," IEEE Transactions on Instrumentation and Measurement, vol. 63, no. 12, pp. 3169-3180, 2014. 
[11] D. L. Donoho and I. M. Johnstone, "Ideal spatial adaptation by wavelet shrinkage," Biometrika, vol. 81, no. 3, pp. 425-455, 1994.

[12] D. L. Donoho, "De-noising by soft-thresholding," IEEE Transactions on Information Theory, vol. 41, no. 3, pp. 613627, 1995.

[13] P. K. Kankar, S. C. Sharma, and S. P. Harsha, "Rolling element bearing fault diagnosis using wavelet transform," Neurocomputing, vol. 74, no. 10, pp. 1638-1645, 2011.

[14] W. Sun, X. Cao, B. Chen, Y. Zhou, Z. Shen, and J. Xiang, "A two-stage vision-based method for measuring the key parameters of ball screws," Precision Engineering, vol. 66, pp. 76-86, 2020.

[15] H. Cao, Y. Lei, and Z. He, "Chatter identification in end milling process using wavelet packets and Hilbert-Huang transform," International Journal of Machine Tools and Manufacture, vol. 69, pp. 11-19, 2013.

[16] A. Wong and X. Y. Wang, "A bayesian residual transform for signal processing," IEEE Access, vol. 3, pp. 709-717, 2015.

[17] L. Meng, J. Xiang, Y. Wang, Y. Jiang, and H. Gao, "A hybrid fault diagnosis method using morphological filter-translation invariant wavelet and improved ensemble empirical mode decomposition," Mechanical Systems and Signal Processing, vol. 51, pp. 101-115, 2015.

[18] N. E. Huang, Z. Shen, S. R. Long et al., "The empirical mode decomposition and the hilbert spectrum for nonlinear and non-stationary time series analysis," Proceedings of the Royal Society of London. Series A: Mathematical, Physical and Engineering Sciences, vol. 454, no. 1971, pp. 903-995, 1998.

[19] Y. Lei, J. Lin, Z. He, and M. J. Zuo, "A review on empirical mode decomposition in fault diagnosis of rotating machinery," Mechanical Systems and Signal Processing, vol. 35, no. 2, pp. 108-126, 2013.

[20] Q. He, P. Li, and F. Kong, "Rolling bearing localized defect evaluation by multiscale signature via empirical mode decomposition," Journal of Vibration and Acoustics, vol. 134, pp. 67-75, 2012.

[21] D. Yu, J. Cheng, and Y. Yang, "Application of EMD method and Hilbert spectrum to the fault diagnosis of roller bearings," Mechanical Systems and Signal Processing, vol. 19, no. 2, pp. 259-270, 2005.

[22] Z. Wu and N. E. Huang, "Ensemble empirical mode decomposition: a noise-assisted data analysis method," Advances in Adaptive Data Analysis, vol. 1, no. 1, pp. 1-41, 2009.

[23] Y. Lei, Z. He, and Y. Zi, "Application of the EEMD method to rotor fault diagnosis of rotating machinery," Mechanical Systems and Signal Processing, vol. 23, no. 4, pp. 1327-1338, 2009.

[24] Y. Lei, N. Li, J. Lin, and S. Wang, "Fault diagnosis of rotating machinery based on an adaptive ensemble empirical mode decomposition," Sensors, vol. 13, no. 12, pp. 16950-16964, 2013.

[25] Y. Lei, Z. He, and Y. Zi, "EEMD method and WNN for fault diagnosis of locomotive roller bearings," Expert Systems with Applications, vol. 38, no. 6, pp. 7334-7341, 2011.

[26] J. P. Dron, F. Bolaers, and L. Rasolofondraibe, "Improvement of the sensitivity of the scalar indicators (crest factor, kurtosis) using a de-noising method by spectral subtraction: application to the detection of defects in ball bearings," Journal of Sound and Vibration, vol. 270, no. 2, pp. 61-73, 2004.

[27] L. Meng, J. Xiang, Y. Zhong, W. Song, and W. L. Song, "Fault diagnosis of rolling bearing based on second generation wavelet denoising and morphological filter," Journal of
Mechanical Science and Technology, vol. 29, no. 8, pp. 31213129, 2015.

[28] D. J. Henderson and C. F. Parmeter, Applied Nonparametric Econometrics, Cambridge University Press, Cambridge, UK, 2015.

[29] I. Horová, J. Koláček, and J. Zelinka, Kernel Smoothing in MATLAB: Theory and Practice of Kernel Smoothing, World Scientific Publishing, Singapore, 2012.

[30] E. A. Nadaraya, "On estimating regression," Theory of Probability \& Its Applications, vol. 9, no. 1, pp. 141-142, 1964.

[31] G. S. Watson, "Smooth regression analysis," Journal of the Indian Society of Agricultural Statistics, vol. 26, pp. 359-372, 1964.

[32] Q. Wu, R. Law, and S. Wu, "Fault diagnosis of car assembly line based on fuzzy wavelet kernel support vector classifier machine and modified genetic algorithm," Expert Systems with Applications, vol. 38, no. 8, pp. 9096-9104, 2011.

[33] Q. Wu, "Car assembly line fault diagnosis model based on triangular fuzzy Gaussian wavelet kernel support vector classifier machine and genetic algorithm," Expert Systems with Applications, vol. 38, no. 12, pp. 14812-14818, 2011.

[34] P. Baraldi, F. Di Maio, P. Turati, and E. Zio, "Robust signal reconstruction for condition monitoring of industrial components via a modified Auto Associative Kernel Regression method," Mechanical Systems and Signal Processing, vol. 61, pp. 29-44, 2015.

[35] H. Takeda, S. Farsiu, and P. Milanfar, "Kernel regression for image processing and reconstruction," IEEE Transactions on Image Processing, vol. 16, no. 2, pp. 349-366, 2007.

[36] D. L. Donoho and I. M. Johnstone, "Adapting to unknown smoothness via wavelet shrinkage," Journal of the American Statistical Association, vol. 90, no. 432, pp. 1200-1224, 1995.

[37] M. D. Buhmann, Radial Basis Functions: Theory and Implementations, Cambridge University Press, Cambridge, UK, 2003.

[38] C. Leys, C. Ley, O. Klein, P. Bernard, and L. Licata, “Detecting outliers: do not use standard deviation around the mean, use absolute deviation around the median," Journal of Experimental Social Psychology, vol. 49, no. 4, pp. 764-766, 2013.

[39] H. Huang and J. Pan, "Speech pitch determination based on Hilbert-Huang transform,” Signal Processing, vol. 86, no. 4, pp. 792-803, 2006.

[40] Y. Lei and M. J. Zuo, "Fault diagnosis of rotating machinery using an improved HHT based on EEMD and sensitive IMFs," Measurement Science and Technology, vol. 20, no. 12, Article ID 125701, 2009.

[41] Y. Wang, J. Xiang, R. Markert, and M. Liang, "Spectral kurtosis for fault detection, diagnosis and prognostics of rotating machines: a review with applications," Mechanical Systems and Signal Processing, vol. 66, pp. 679-698, 2015.

[42] H. Konstantin-Hansen and H. Herlufsen, "Envelope and cepstrum analyses for machinery fault identification," J. Sound Vib, vol. 44, pp. 10-12, 2010.

[43] Case Western Reserve University Bearing Data Center Website, 〈http://csegroups.case.edu/bearingdatacenter/home〉.

[44] J. Xiang, Y. Zhong, and H. Gao, "Rolling element bearing fault detection using PPCA and spectral kurtosis," Measurement, vol. 75, pp. 180-191, 2015.

[45] J. Xiang and Y. Zhong, "A fault detection strategy using the enhancement ensemble empirical mode decomposition and random decrement technique," Microelectronics Reliability, vol. 75, pp. 317-326, 2017. 